\title{
Simulation of the phase diagram of magnetic vortices in two-dimensional superconductors: Evidence for vortex chain formation
}

\author{
$\mathrm{X} \mathrm{B} \mathrm{Xu},{ }^{1,}{ }^{*} \mathrm{H}$ Fangohr, ${ }^{2} \mathrm{M} \mathrm{Gu},{ }^{1} \mathrm{~W}$ Chen, ${ }^{1} \mathrm{Z} \mathrm{H}$ Wang, ${ }^{1}$ F Zhou, ${ }^{1}$ D Q Shi, ${ }^{3}$ and S X Dou \\ ${ }^{1}$ Center for Superconducting Physics and Materials, \\ National Laboratory of Solid State Microstructures and Department of physics, \\ Nanjing University, Nanjing 210093, P. R. China \\ ${ }^{2}$ Engineering and the Environment, University of Southampton, Southampton SO17 1BJ, United Kingdom \\ ${ }^{3}$ Institute for Superconducting and Electronic Materials, \\ University of Wollongong, NSW 2522, Australia
}

\begin{abstract}
We study the superconducting vortex states induced by the interplay of long-range Pearl repulsion and short-range intervortex attraction using Langevin dynamics simulation. We show that at low temperature the vortices form an ordered Abrikosov lattice both in low and high fields. The vortices show distinctive modulated structures at intermediate fields depending on the effective intervortex attraction: ordered vortex chain and Kogame-like vortex structures for weak attraction; bubble, stripe and antibubble lattices for strong attraction. Moreover, in the regime of the chain state, the vortices display structural transitions from chain to labyrinthine (or disordered chain) and/or to disordered state depending on the strength of disorders.
\end{abstract}

PACS numbers: $74.25 . \mathrm{Dw}, 74.25 . \mathrm{Uv}$

\section{INTRODUCTION}

Superconducting vortex states have proved to be a rich and attractive research field. In 1954 Abrikosov solved the Ginzburg-Landau (GL) equations in an applied magnetic field for the GL parameter $\kappa>1 / \sqrt{2}$, and found his famous vortex solution where the superconducting order parameter contains a periodic lattice of zeros. This leads to a new mixed Abrikosov vortex phase between the Meissner state and the normal state [1]. In real superconductors the equilibrium vortex structures are controlled by the competition between vortex-vortex interactions and vortex-disorder interactions [2]. As the disorders become important, the ordered Abrikosov mixed state will change into disordered liquid due to thermal fluctuations, or into glass states due to pinning [3-5].

Strikingly, unconventional vortex pattern formation was recently discovered in multiband type-II superconductors, such as $\mathrm{MgB}_{2}[6]$ and $\mathrm{Sr}_{2} \mathrm{RuO}_{4}[7]$ and ironpnictide materials $[8,9]$, where vortices display chainlike (and/or stripelike) structures in low fields. Although large amount of experimental and theoretical efforts in this field has been made [10-18], the nature of the vortex clustering in multiband superconductors is still an intensely controversial issue now $[17,19-22]$. In principle, the vortex cluster formation means that there might exist some type of attraction between vortices in addition to repulsion. This vortex attraction may have various possible origins: (I) Twin boundaries can act as a source of one-dimensional strong pinning and thus can produce vortex chains or stripes [8]. (II) For the superconductors with $\kappa \sim 1 / \sqrt{2}$ such as Nb [4], the non-local electrodynamics induced by the spatially varying order parameter produces a relationship between supercurrents and vector potential. This may result in an oscillating vortex-vortex potential with attractive tail at larger distances [23]. (III) For moderately anisotropic superconductors like YB$\mathrm{CO}$, it has been demonstrated that the field changes sign in some local regions in the plane perpendicular to the flux-line axis under a tilted magnetic field [24-26], leading to intervortex attraction in those regions. (IV) For the layered superconductors with very weak inter-layer coupling, the Josephson Vortices (JV) will appear at much lower field near $\mathrm{H}_{\mathrm{c} 1}$ [25]. The PV are attracted and trapped by JV and thus form chains [27, 28]. Besides, for layered superconductors, the intervortex attractions can also be induced by thermal fluctuations [29,30], or impurities [31]. (V) For multiband or multicomponent superconductors, the intervortex interaction is determined by the competition between the multiple fundamental length scales in GL theory $[12,16,19]$ : leads to attractive interaction at a long range; yet the repulsive interaction is still dominating at a short range due to intercurrent and electromagnetic interaction. However, numerical simulations reveal that the nonmonotonic interaction potential with long-range attraction will result in the bubble-like rather than stripe-like vortex ground state [32]. In presence of the quenched disorders, vortices form metastable and disordered fragmental structures in practical superconducting systems. Thus, it is still a long-standing challenge to understand the nature of vortex chain state, especially in isotropic multiband or multicomponent superconductors. In additional, the followed two questions are also open for these chain-forming systems: (1) whether are there additional new phases? (2) what are the effects of thermal fluctuations or quenched disorders on vortex chain?

In fact, the above-mentioned experimental observations have given two important clues to study the physics underlying vortex chain. First, it was shown that chainlike vortex distributions are of long-range order both in 


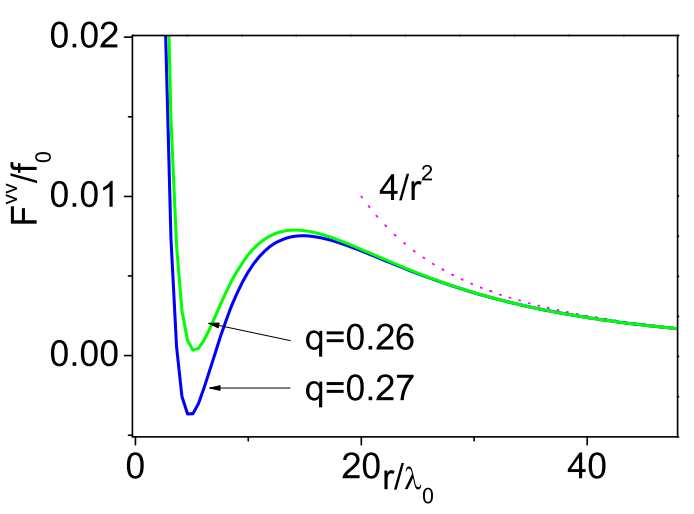

FIG. 1: (Color online) Vortex-vortex interaction force as function of the inter-vortex distance for different q. The magenta dot line shows the limiting expression at large distances. The limit at small distances is $F^{v v}(r) \approx 1 / r$, for $r \ll \lambda$ (not shown here for simplicity). anisotropic superconductors such as YBCO [26], and in isotropic ones such as $\mathrm{MgB}_{2}$ [6]. This means that the vortex interaction in a long range is of repulsive rather than attractive for the formation of vortex chains. Here, we consider the long-range Pearl repulsion as a candidate $[33,34]$. It is produced by the stray field near the surface outside the samples and should be important for thin samples [25, 35]. Second, the chainlike clustering structure of vortices in superconductors is indicative of intervortex attraction. For multcomponent or multiband superconductors, it has been found that the London penetration length $\lambda$ can be comparable to the coherence length $\xi$ at some material parameter space $[13,19]$. In this case, the attraction due to the overlapping of vortex cores (reducing the condensation energy) becomes relevant in addition to the repulsion (induced be current-current and electromagnetic interactions). Based on these ideas, we propose a phenomenological intervortex interaction model with Pearl repulsion and core-core attraction to study vortex states of chainforming systems by Langevin dynamics. We numerically demonstrate that the vortices form ordered chains due to the competing vortex-vortex interactions. Moreover, we show the formation of other interesting structures such as Kogame-like vortex domains depending on the magnitude of field, bubble and stripe and antibubble lattices on the effective intervortex attraction, and labyrinth on the strength of disorders.

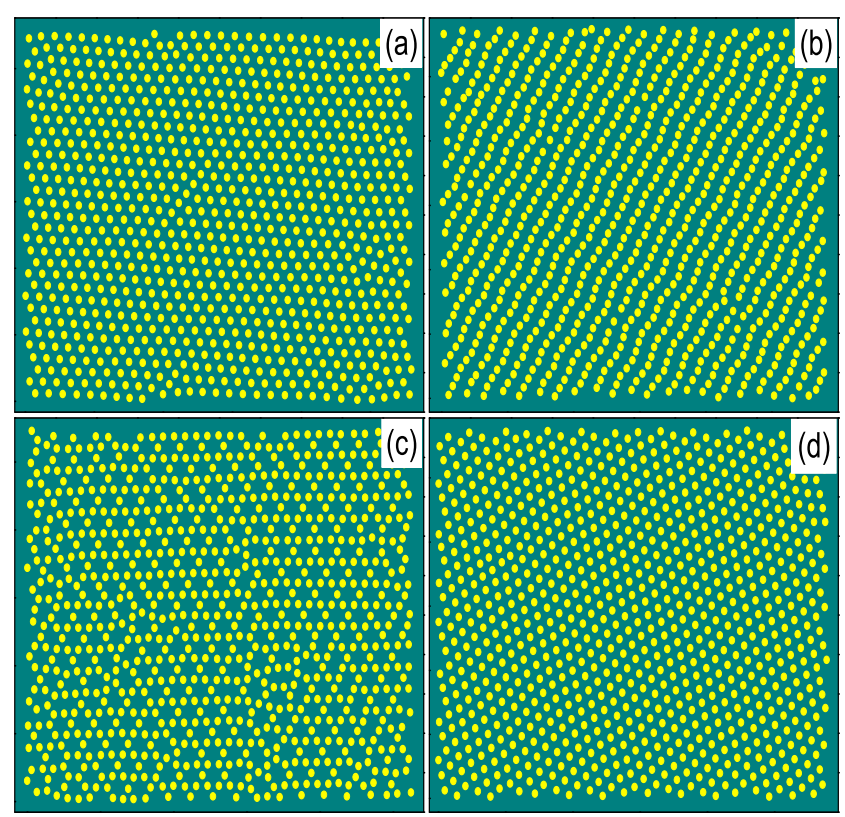

FIG. 2: (Color online) Static vortex configurations for different densities at zero temperature. Panel (a) shows the low-density hexagonal lattice for $B=0.05 B_{c 20}$. Panels (b) and (c) show the ordered vortex chains for $B=0.15 B_{c 20}$ and the Kogamelike vortex structures for $B=0.22 B_{c 20}$, respectively. Panel (d) shows the high-density hexagonal lattice for $B=0.3 B_{c 20}$.

\section{SIMULATION}

The overdamped Langevin equation of motion for a vortex at position $\mathbf{r}_{i}$ is [36]

$$
\mathbf{F}_{i}=\sum_{j \neq i}^{N_{\mathrm{v}}} \mathbf{F}^{\mathrm{vv}}\left(\mathbf{r}_{i}-\mathbf{r}_{j}\right)+\sum_{k}^{N_{\mathrm{p}}} \mathbf{F}^{\mathrm{vp}}\left(\mathbf{r}_{i}-\mathbf{r}_{k}^{\mathrm{p}}\right)+\mathbf{F}_{i}^{\mathrm{T}}=\eta \frac{d \mathbf{r}_{i}}{d t}
$$

where $\mathbf{F}_{i}$ is the total force acting on vortex $i, \mathbf{F}^{\mathbf{v v}}$ and $\mathbf{F}^{\mathrm{vp}}$ are the forces due to vortex-vortex and vortex-pin interactions, $\mathbf{F}^{\mathrm{T}}$ is the thermal stochastic force, $\eta$ is the Bardeen-Stephen friction coefficient, $N_{\mathrm{v}}$ is the number of vortices, $N_{\mathrm{p}}$ is the number of pinning centers and $\mathbf{r}_{k}^{\mathrm{p}}$ is the position of the $k$ th pinning center. The effective interaction between two vortices separated by $\mathbf{r}$ (shown in Fig. 1) is

$F^{\mathrm{vv}}(\mathbf{r})=\frac{\phi_{0}^{2}}{8 \pi \Lambda^{2} \mu_{0}}\left[H_{1}\left(\frac{r}{\Lambda}\right)-Y_{1}\left(\frac{r}{\Lambda}\right)-\frac{2}{\pi}-q \sqrt{\frac{\xi}{r}} \exp \left(-\frac{r}{\xi}\right)\right]$

where $\phi_{0}$ is the flux quantum, $\Lambda=2 \lambda^{2} / s, s$ is the thickness of the superconducting films, $\mu_{0}$ is the vacuum permeability, $\mathrm{H}_{1}$ and $\mathrm{Y}_{1}$ are the Struve and Bessel function$\mathrm{s}$, and $r$ is the distance between vortices. The first three 

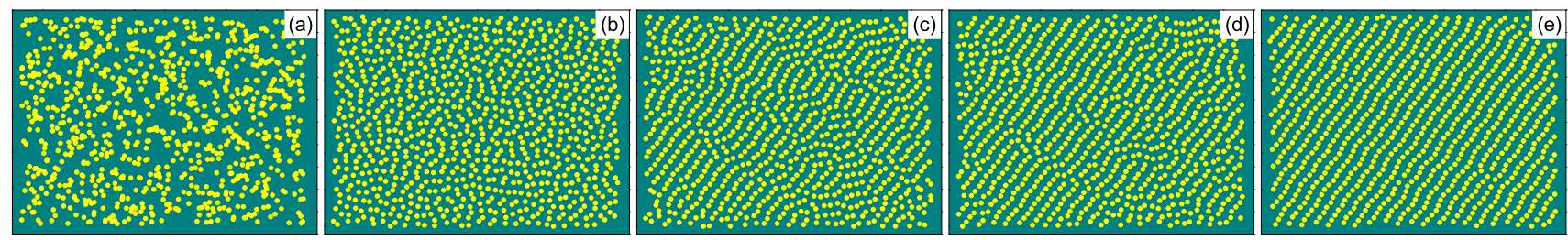

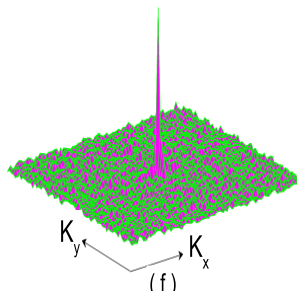

(f)



(g)

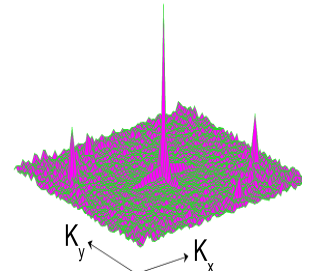

(h)

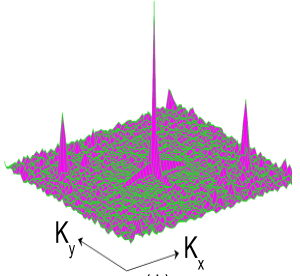

(i)

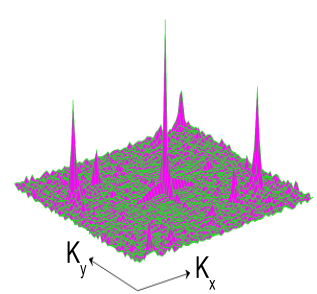

(j)

FIG. 3: (Color online) Time evolution of the formation of the ordered vortex chains, starting from a random disordered state, as shown in (a), and the corresponding structure factors with $B=0.15 B_{c 20}$ and $T=0$. The vortex configurations: (b) $t=6$, (c) $t=92$, (d) $t=108$, (e) $t=386$. The corresponding structure factor $\mathrm{S}(\mathrm{k})$ : (f) $t=0$, (g) $t=6$, (h) $t=92$, (i) $t=108$, and (j) $t=386$.
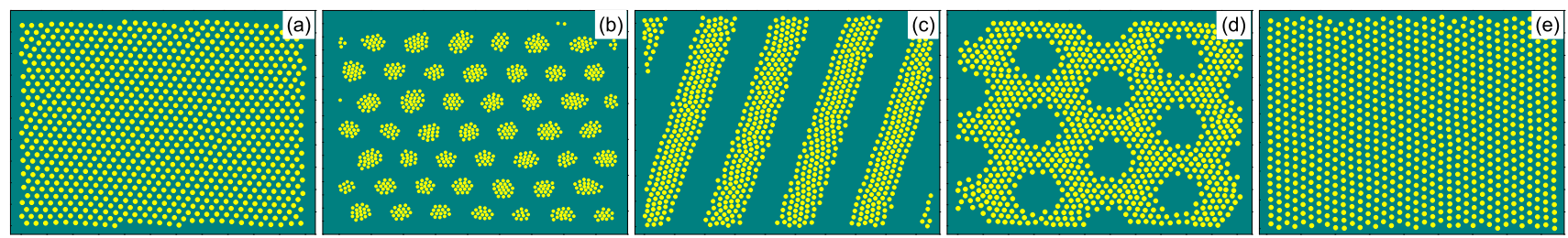

FIG. 4: (Color online) Static vortex configurations for $q=0.27$ at zero temperature for different densities: panel (a) ordered low-field lattice for $B=0.03 B_{c 20}$, panel (b) bubble lattice for $B=0.14 B_{c 20}$, (c) stripe lattice for $B=0.28 B_{c 20}$, panel (d) antibubble lattice for $B=0.3 B_{c 20}$, panel (e) ordered high-field lattice for $B=0.5 B_{c 20}$.

terms show a long-range repulsion between the vortices for thin samples $[33,37,38]$. The fourth term is the intervortex attraction by considering the core-core attraction $V_{\text {attra }} \sim \exp (-r / \xi) / \sqrt{r / \xi}$ at a larger distance (corresponding to low magnetic fields in which vortex chains were observed) [4]. The parameter $q$ reflects the relative strength of attraction to repulsion. We employ periodic boundary conditions and cut off the vortex-vortex interaction potential smoothly $[39,40]$. A pinning center at position $\mathbf{r}_{k}^{\mathrm{p}}$ exerts an attractive force on the vortex at position $\mathrm{r}_{i}: \mathbf{F}^{\mathrm{vp}}\left(\mathbf{r}_{i}-\mathbf{r}_{k}^{\mathrm{p}}\right)=-f_{\mathrm{pv}}\left(r_{i k} / r_{\mathrm{p}}\right) \exp \left(-\left(r_{i k} / r_{\mathrm{p}}\right)^{2}\right) \hat{\mathbf{r}}_{i k}$, where $f_{\mathrm{pv}}$ tunes the strength of this force and $r_{\mathrm{p}}$ determines its range. We use $f_{\mathrm{pv}} \propto B_{\mathrm{c} 2}^{2}\left(1-B / B_{\mathrm{c} 2}\right) \xi^{2} / \kappa^{2}$ as the core pinning [41], where $\kappa=\lambda / \xi$. The thermal fluctuation force has properties $\left\langle F_{i}^{\mathrm{T}}\right\rangle=0$ and $\left\langle F_{i}^{\mathrm{T}}(t) F_{j}^{\mathrm{T}}\left(t^{\prime}\right)\right\rangle=$ $2 \eta k_{\mathrm{B}} T \delta_{i j} \delta\left(t-t^{\prime}\right)$ at a given temperature $T$. We normalize lengths by $\lambda$, forces by $f_{0}=\phi_{0}^{2} /\left(8 \pi \Lambda^{2} \mu_{0}\right)$ and time by $\tau_{0}=\Lambda \eta / f_{0}$. All quantities shown here are expressed in these simulation units. The equation of motion is integrated by an Euler scheme with a normalized time step of
$\Delta t=0.0005$ [40]. The total number of vortices $N_{\mathrm{v}}=900$ is used in the calculations. For larger systems, similar results are observed. We employ $q=0.26$ unless specified otherwise, $r_{p}=0.15 \lambda, \xi=1000 \AA, \lambda=200 \AA, s=40 \AA$, and $\eta=1.4 \times 10^{-17} \mathrm{~kg} / \mathrm{s}$. The vortices are randomly distributed for the initial vortex state.

\section{VORTEX STATES AND VORTEX CHAIN FORMATION AT ZERO TEMPERATURE}

We first consider the static vortex configurations, illustrated in Fig. 2, obtained at different vortex densities for zero temperature. At low vortex densities, it can be seen that the vortices form the hexagonal Abrikosov lattice shown in Fig. 2(a). It is clear that this low-field equilibrium structure is a direct result of the long-range repulsive tail in our model system due to a large average distance between vortices. For increasing vortex density, the vortex system exhibits ordered vortex chains, as 
seen in Fig. 2(b). The formation of vortex chain structure marks that the average vortex distance is in the region of repulsive shoulder: the system energy is lowered by bringing vortices together on the shoulders and thus reducing the number of nearest neighbors. With further increasing density some vortices will move away from lines and enter into the regions between lines because of the enhanced vortex-vortex interactions. This leads to one novel type of vortex structure, domains with Kogame lattice where each vortex has four neighboring ones, as illustrated in Fig. 2(c). These Kogamelike patterns persist up to $\mathrm{B}=0.25 \mathrm{~B}_{\mathrm{c} 2}$, when a high-density ordered Abrikosov lattice occurs due to the dominating short-range repulsion, as shown in Fig. 2(d).

We next focus on the formation of vortex chain. For the sake of simplicity, we leave the evolution dynamics of the Kogamelike vortex state to a future study. We choose a random initial configuration for vortex positions. However, the final equilibrium vortex chain pattern does not depend on the particular choice of the initial configuration. Illustrated in Fig. 3 are the real space images and the corresponding structure factors, $S(\mathbf{k})=\left|\sum_{i=1}^{N_{v}} \exp \left[i \mathbf{k} \cdot \mathbf{r}_{i}\right]\right|^{2} / N_{v}$, which are used to quantify changes in the vortex structure at different times. It can be seen that the time evolution is characterized by three major stages: (i) appearance of homogeneously disordered configurations through the amplifications of the initial fluctuations [Fig. 3(b)]; (ii) formation of disordered chain domains [Fig. 3(c), (d)]; and (iii) slow relaxation of disordered chains to smectic chains [Fig. 3(e)]. In the first stage, the vortex motion is mainly controlled be the strongly short-range repulsion. Thus, the vortices move away from each other with a relatively fast speed, and form homogeneous vortex structure. The corresponding structure factors $\mathrm{S}(\mathrm{k})$ shows one central peak, indicating the absence of ordering [Fig. 3(g)]. In the intermediate stage, the long-range repulsion becomes important because of the increment of average distance between vortices. The competition between intervortex repulsion and attraction manifesties itself as the emergence of chainlike domain structure. Correspondingly some weak peaks occur, which are definitely larger than the background, as shown in Fig 3(h) and (i). In the final stage, the vortex configuration is determined by the long-range repulsion. In this case, the disordered chains become ordered with a slow relaxation due to the weak vortex-vortex interactions. The occurrence of two peaks in structure factors is due to the smectic ordering of the chain structures [Fig. 3(j)].

We then study the vortex states for a larger $q(=0.27)$, corresponding to the minimum of the intervortex interation appears to be below zero level in Fig. 1. Fig. 4 shows the equilibrium vortex structures for different vortex densities at zero temperature. For both low and high vortex densities, the vortices form ordered lattice due to the dominating intervortex repulsion (Fig. 4(a) and Fig. 4(e)). While for intermediate vortex densities, the vortex system displays three kinds of ordered vortex domain structures with increasing vortex density: bubble (Fig. 4(b)), stripe (Fig. 4(c)) and antibubble (Fig. $4(\mathrm{~d})$ ). It has been known that the formation of these modulated structures originates from the competition between short-range attraction and long-range repulsion, observed in wide physical systems [42]. The numerical vortex structures including vortex bubble and stripe are in good agreement with the experimental observations in low- $\kappa$ type II superconductors [4, 43]. Also, for anisotropic superconductors, the theoretical calculation and analysis based on London theory and LowrenceDoniach theory has been shown that multiquanta vortex lattices will occur through changing titling angles of magnetic fields (equivalent to changing the value of $q$ in this simulation) [25]. This suggests that our model is useful to study the vortex states for both low- $\kappa$ type II superconductors and anisotropic superconductors, in addition to multiband or multicomponent superconductors [12].

\section{EFFECTS OF FILM THICKNESS AND DISORDERS ON VORTEX STATES}

We next studied the effect of film thickness on vortex states. In Fig. 5 we show the typical vortex structures for various film thickness at fixed B and q. A decrement of film thickness is equivalent to an increment of repulsion between vortices, or to an decrement of attraction between vortices. Thus, with decreasing film thickness, the vortex states change from intermediate-mixed states, i.e., ordered antibubbles (Fig. 5(a)), stripes (Fig. 5(b)) and bubbles (Fig. 5(c)), to the Abrikosov lattice (Fig. 5(d)). In other words, superconductors that are type-I or socalled type-1.5 in bulk, show type-II behavior in thin films since they have an effective $\kappa$ much larger than in bulk $[16,34,44]$. That is, our simulations concur with the Pearl theory[34].

We now consider the effects of thermal fluctuations and pinning on vortex chain state. For relatively smal1 fluctuations, the vortex system shows ordered chains due to dominating vortex-vortex interactions, as shown in Fig. 6(a). As the fluctuations become large enough, the vortex line structure will be completely destroyed, shown in Fig. 6(b). On the other hand, the quenched disorder$\mathrm{s}$ in systems can also induce the structure changes from ordered chains to disordered states. Clearly, this depend$\mathrm{s}$ on the competition between vortex elastic energy and pinning energy. For a relative small pinning strength, we find that vortices form a labyrinthine structure, see Fig. 6(c). For strong vortex pinning, the vortex system will be trapped into a long-lived metastable disordered state due to a longer relaxation time, see Fig. 6(d). These simulations might explain why the disordered chains or stripes were frequently observed $[10,11,15]$, while the 


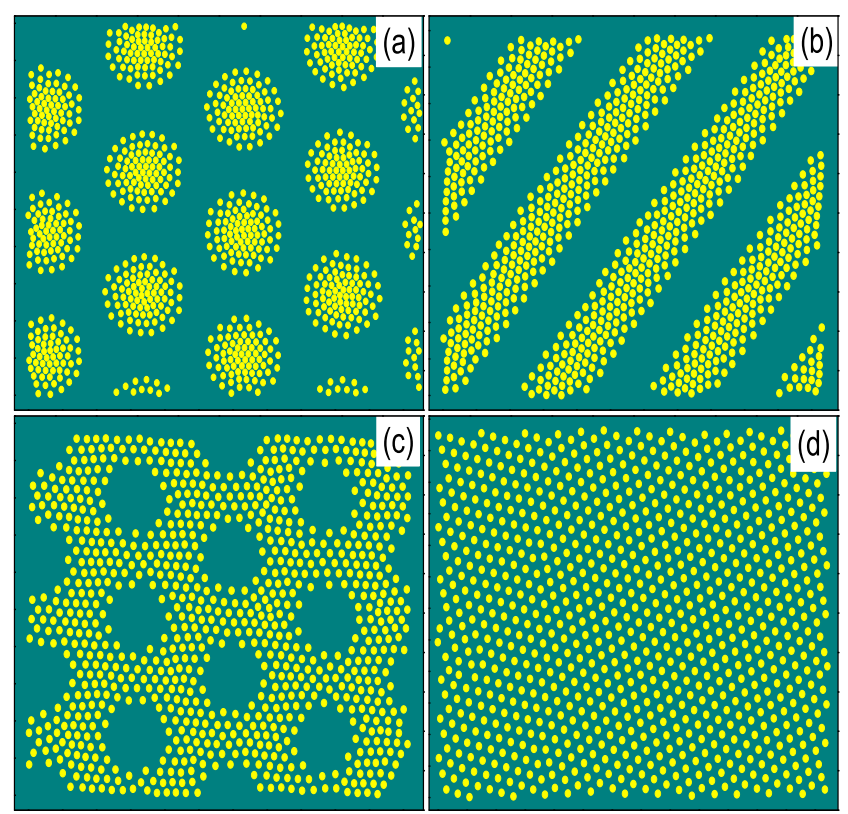

FIG. 5: (Color online) Static vortex configurations for different $s$ for $q=0.27$ and $B=0.3 B_{c 20}$ at zero temperature: panel (a) ordered bubbles for $s=0.215 \lambda$, panel (b) ordered stripes for $s=0.21 \lambda$, panel (c) ordered antibubbles for $s=0.2 \lambda$, panel (d) ordered lattice for $s=0.05 \lambda$.

ordered line state was seldom found in experiments.

\section{PHASE DIAGRAM}

We summarize the transitions between different vortex states, identified by their structure factor $\mathrm{S}(\mathrm{k})$, as functions of vortex density and temperature in the phase diagram displayed in Fig. 7. This is the main result in our report. In the low-temperature regime, where thermal fluctuations are weak, with increasing density the vortex system shows low-density Abrikosov lattice $\left(\mathrm{B} \lesssim 0.1 \mathrm{~B}_{\mathrm{c} 2}\right)$, ordered chain state $\left(\mathrm{B}=0.1 \sim 0.19 \mathrm{~B}_{\mathrm{c} 2}\right)$, Kogamelike vortex state $\left(\mathrm{B}=0.19 \sim 0.25 \mathrm{~B}_{\mathrm{c} 2}\right)$, and high-density Abrikosov lattice $\left(\mathrm{B} \gtrsim 0.25 \mathrm{~B}_{\mathrm{c} 2}\right)$. The boundaries between these phases are the direct consequences of the competition between long-range and short-range repulsion (or two characteristic length scales). In the hightemperature regime, thermal fluctuations become important. The vortex phases transit from ordered solid to disordered liquid state with increasing temperature. The phase boundaries result from the competition between vortex elastic energy and thermal energy.

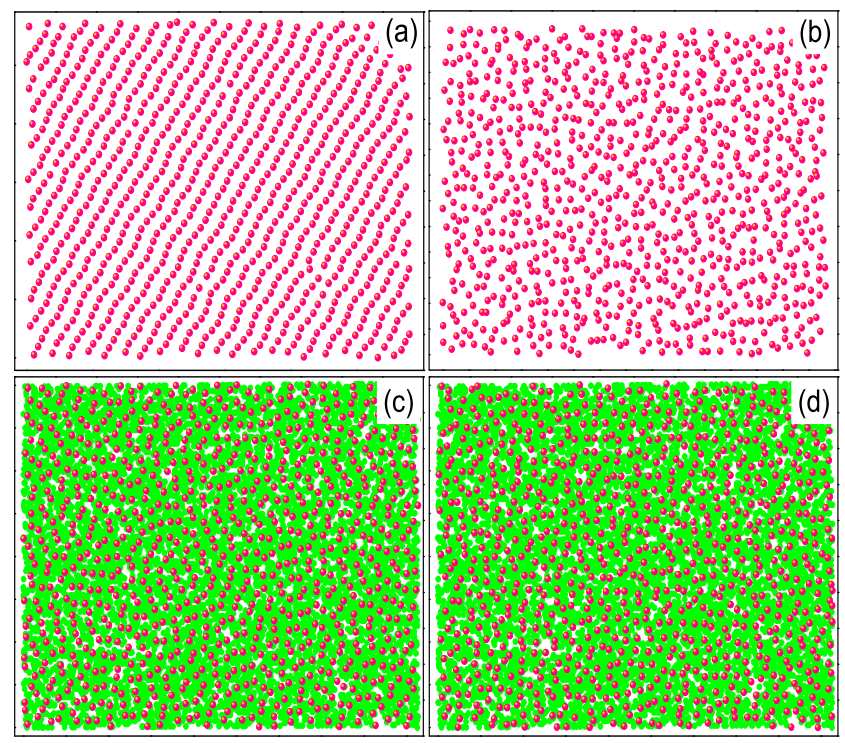

FIG. 6: (Color online) Effect of disorders on the configurations of vortices (pink solid circles) at $B=0.15 B_{c 20}$, including thermal fluctuations: (a) $T=0.1$, (b) $T=0.7$; and pinning centers (green solid circles, $N_{p}=10 N_{v}$ ): (c) $f_{\mathrm{pv}}=0.4, T=0$, (d) $f_{\mathrm{pv}}=4, T=0$.

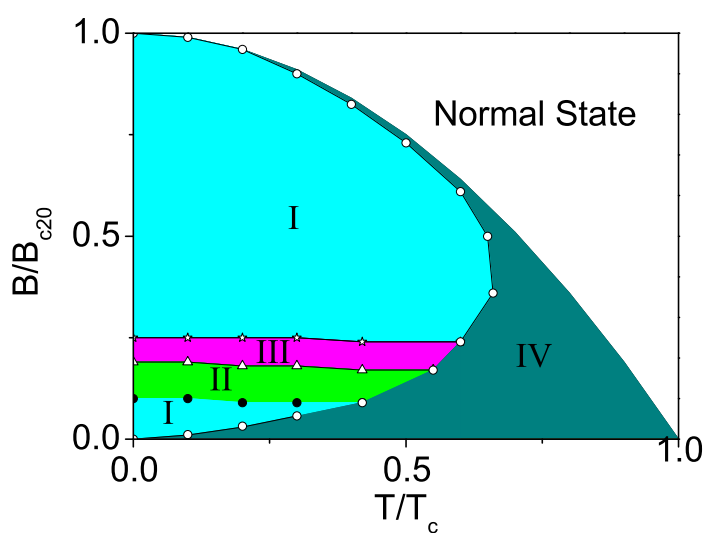

FIG. 7: (Color online) Phase diagram of vortex matter as function of $\mathrm{B}$ and $\mathrm{T}$. The phases are hexagonally ordered vortex lattice (I), ordered vortex chains (II), Kogamelike vortex phase (III), and vortex liquid (IV).

\section{SUMMARY}

In conclusion, we propose a phenomenological vortexvortex interaction model with long-range Pearl repulsion and short-range attraction to study the vortex states of chain-forming vortex systems. We find a rich phase diagram for the clean vortex system which includes Abrikosov, ordered chain, Kogame and liquid phases. We 
also find that the ordered vortex chain phase transits into a disordered chain and disordered glass phases in the presence of impurities. Besides, for enhanced intervortex attraction, we show that the vortices form ordered lattice both in low and high vortex densities, and bubble and stripe and antibubble lattice at intermediate vortex densities. Our results are in agreement with experiments.

X.B. thank Hai-Hu Wen for helpful discussions. This work was supported by grants from the Ministry of Science and Technology (MOST) 973 Program of China (No. 2011CBA00107, No. 2011CB933400 and No. 2008CB601003), the National Science Foundation (NSF) of China (No. 91021003 and No. 10674060), the Project Funded by the Priority Academic Program Development of Jiangsu Higher Education Institutions (PAPD).

* Corresponding author. Email: xxb@nju.edu.cn

[1] A. A. Abrikosov, Zh. Eksp. Teor. Fiz. 32, 1442 (1957) [Sov. Phys. JETP 5, 1174 (1957)].

[2] G. Blatter, M. V. Feigel'man, V. B. Geshkenbein, A. I. Larkin, and V. M. Vinokur, Rev. Mod. Phys. 66, 1125 (1994).

[3] see, e.g., T. Giamarchi and S. Bhattacharya, arXiv: cond-mat/0111052v1.

[4] E. H. Brandt, Rep. Prog. Phys. 58, 1465 (1995).

[5] B. Rosenstein, and D. P. Li, Rev. Mod. Phys. 82, 109 (2010).

[6] J. Gutierrez, B. Raes, A. V. Silhanek, L. J. Li, N. D. Zhigadlo, J. Karpinski, J. Tempere, and V. V. Moshchalkov, Phys. Rev. B 85, 094511 (2012).

[7] P. J. Curran, V. V. Khotkevych, S. J. Bending, A. S. Gibbs, S. L. Lee, and A. P. Mackenzie, Phys. Rev. B 84, 104507 (2011)

[8] H. Yang, B. Shen, Z. Y. Wang, L. Shan, C. Ren, and Hai-Hu Wen, Phys. Rev. B 85, 014524 (2012).

[9] L. J. Li, T. Nishio, Z. A. Xu, and V. V. Moshchalkov, Phys. Rev. B 83, 224522 (2011).

[10] T. Nishio, V. H. Dao, Q. Chen, L. F. Chibotaru, K. Kadowaki, and V. V. Moshchalkov, Phys. Rev. B 81, 020506(R) (2010); V. Moshchalkov, M. Menghini, T. Nishio, Q. H. Chen, A. V. Silhanek, V. H. Dao, L. F. Chibotaru, N. D. Zhigadlo, and J. Karpinski, Phys. Rev. Lett. 102, 117001 (2009).

[11] V. O. Dolocan, C. Veauvy, F. Servant, P. Lejay, K. Hasselbach, Y. Liu, and D. Mailly, Phys. Rev. Lett. 95, 097004 (2005); P. G. Björnsson, Y. Maeno, M. E. Huber, and K. A. Moler, Phys. Rev. B 72, 012504 (2005); V. O. Dolocan, P. Lejay, D. Mailly, and K. Hasselbach, Phys. Rev. B 74, 144505 (2006).

[12] E. Babaev and M. Speight, Phys. Rev. B 72, 180502(R) (2005); E. Babaev, J. Carlström, and M. Speight, Phys. Rev. Lett. 105, 067003 (2010); E. Babaev, J. Carlström, and M. Speight, Phys. Rev. Lett. 105, 067003 (2010); J. Carlström, J. Garaud, and E. Babaev, Phys. Rev. B 84, 134518 (2011)

[13] E. Babaev, J. Carlström, Physica C 470, 717 (2010).

[14] A. Chaves, L. Komendová, M. V. Milošević, J. S. Andrade Jr., G. A. Farias, and F. M. Peeters, Phys. Rev. B
83, $214523(2011)$

[15] Shi-Zhen Lin and Xiao Hu, Phys. Rev. B 84, 214505 (2011).

[16] J. Garaud, D. F. Agterberg, and E. Babaev, Phys. Rev. B 86, 060513(R) (2012).

[17] V. G. Kogan and J. Schmalian, Phys. Rev. B 83, 054515 (2011).

[18] B. Rosenstein, I. Shapiro, and B. Ya. Shapiro, Phys. Rev. B 83, 064512 (2011).

[19] E. Babaev and M. Silaev, Phys. Rev. B 86, 016501 (2012).

[20] E. H. Brandt and M. P. Das, J. Supercond. Nov. Magn. 24, 57 (2011).

[21] A. A. Shanenko, M. V. Milošević, F. M. Peeters, and A. V. Vagov, Phys. Rev. Lett. 106, 047005 (2011).

[22] V. G. Kogan and J. Schmalian, Phys. Rev. B 86, 016502 (2012).

[23] A. E. Jacobs, Phys. Rev. B 4, 3029 (1971).

[24] L. L. Daemen, L. J. Campbell, and V. G. Kogan, Phys. Rev. B 46, 3631 (1992).

[25] A. V. Samokhvalov, D. A. Savinov, A. S. Mel'nikov, and A. I. Buzdin, Phys. Rev. B 82, 104511 (2010).

[26] J. A. Herbsommer, G. Nieva, and J. Luzuriaga, Phys. Rev. B 62, 3534 (2000); P. L. Gammel, D. J. Bishop, J. P. Rice, and D. M. Ginsberg, Phys. Rev. Lett. 68, 3343 (1992).

[27] A. E. Koshelev, Phys. Rev. Lett. 83, 187 (1999).

[28] Y. Segev, Y. Myasoedov, E. Zeldov, T. Tamegai, G. P. Mikitik, and E. H. Brandt, Phys. Rev. Lett. 107, 247001 (2011); A. N. Grigorenko, S. J. Bending, I. V. Grigorieva, A. E. Koshelev, T. Tamegai, and S. Ooi, Phys. Rev. Lett. 94, 067001 (2005); A. Grigorenko, S. Bending, T. Tamegai, S. Ooi, and M. Henini, Nature 414, 728 (2001); C. A. Bolle, P. L. Gammel, D. G. Grier, C. A. Murray, D. J. Bishop, D. B. Mitzi, and A. Kapitulnik, Phys. Rev. Lett. 66, 112 (1991).

[29] E. H. Brandt, R. G. Mints, and I. B. Snapiro, Phys. Rev. Lett. 76, 827 (1996).

[30] G. Blatter, and V. B. Geshkenbein, Phys. Rev. Lett. 77, 4958 (1996).

[31] S. Mukherji, and T. Nattermann, Phys. Rev. Lett. 79, 139 (1997).

[32] C. Reichhardt, J. Drocco, C. J. Olson Reichhardt, A. R. Bishop, Physica C 479, 15 (2012).

[33] J. Pearl, Appl. Phys. Lett. 5, 65 (1964).

[34] J. Pearl, J. Appl. Phys. 37, 4139 (1966).

[35] A. I. Buzdin, A. S. Mel'nikov, A. V. Samokhvalov, T. Akashi, T. Masui, T. Matsuda, S. Tajima, H. Tadatomo, and A. Tonomura, Phys. Rev. B 79, 094510 (2009).

[36] X. B. Xu, H. Fangohr, X. N. Xu, M. Gu, Z. H. Wang, S. M. Ji, S. Y. Ding, D. Q. Shi, and S. X. Dou, Phys. Rev. Lett. 101, 147002 (2008).

[37] J. R. Clem, Phys. Rev. B 43, 7837 (1991).

[38] V. G. Kogan, Phys. Rev. B 75, 064514 (2007).

[39] H. Fangohr, A. Price, S. Cox, P. A. J. de Groot, G. J. Daniell, and K. S. Thomas, J. Comput. Phys. 162, $372(2000)$.

[40] H. Fangohr, Simon J. Cox,, and P. A. J. de Groot, Phys. Rev. B 64, 064505 (2001).

[41] H. J. Jensen, A. Brass, A. C. Shi, and A. J. Berlinsky, Phys. Rev. B 41, 6394 (1990).

[42] B. P. Stojković, Z. G. Yu, A. R. Bishop, A. H. Castro Neto, and Niels Grønbech-Jensen, Phys. Rev. Lett. 82, 4679 (1999); K. Nelissen, B. Partoens, F. M. Peeters, 
Phys. Rev. E 71, 066204 (2005); C. Reichhardt, C. J. Olson Reichhardt, and A. R. Bishop, Physica C 460-462, 1178 (2007); C. Reichhardt, C. J. Olson Reichhardt, I. Martin, and A. R. Bishop, Phys. Rev. Lett. 90, 026401 (2003); C. Reichhardt, C. J. Olson, I. Martin, and A. R. Bishop, Europhys. Lett. 61, 221 (2003); C. J. Olson Reichhardt, C. Reichhardt, I. Martin, and A. R. Bishop, Physica D 193, 303 (2004); H. J. Zhao, V. R. Misko, and F. M. Peeters, Phys. Rev. E 88, 022914 (2013); H. J. Zhao, V. R. Misko, and F. M. Peeters, Physica C 130-
133, 479 (2012).

[43] X. B. Xu, H. Fangohr, S. Y. Ding, F. Zhou, X. N. Xu, Z. H. Wang, M. Gu, D. Q. Shi, and S. X. Dou, Phys. Rev. B 83, 014501 (2011); X. B. Xu, H. Fangohr, Z. H. Wang, M. Gu, S. L. Liu, D. Q. Shi, and S. X. Dou, Phys. Rev. B 84, 014515 (2011).

[44] R. P. Huebener, and J. R. Clem, Rev. Mod. Phys. 409, 46 (1974). 